\title{
Equal opportunity for all? An analysis of race and ethnicity in fertility preservation in New York City
}

\author{
Paxton E. Voigt ${ }^{1,2}$ (D) Jennifer K. Blakemore ${ }^{3} \cdot{\text { David } \text { McCulloh }^{3} \cdot \text { M. Elizabeth Fino }}^{3}$
}

Received: 30 April 2020 / Accepted: 13 October 2020 / Published online: 21 October 2020

(C) Springer Science+Business Media, LLC, part of Springer Nature 2020

\begin{abstract}
Purpose To compare the racial and ethnic make-up of patients who accessed medically indicated fertility preservation services (MIFP) against the overall racial diversity (including Hispanic origin) across women of reproductive age diagnosed with cancer in New York City (NYC).

Methods All patients who completed at least one MIFP between January 2017 and December 2018 were reviewed. Race was self-reported. A calculation of the expected racial distribution across women of reproductive age with cancer in NYC was determined using the most recent NYC census data. Statistical analysis included chi-square goodness of fit and test for independence and Kruskal-Wallis $H$ test, with $p<0.05$ considered significant.

Results A total of 107 patients who accessed MIFP were included. A total of $55(51.4 \%)$ identified as White, $3(2.8 \%)$ as Black, $13(12.2 \%)$ as Asian, $6(5.6 \%)$ as Hispanic, $3(2.8 \%)$ as other, and $27(25.2 \%)$ did not report. A total of 78.5\% of patients had insurance. There was no significant difference in racial distribution by cancer type $(p=0.255)$. A subgroup analysis excluding the BRCA+ patients and races not reported by the census $(n=69)$ was then performed, showing a statistically significant difference between observed $(\mathrm{O})$ and expected $(\mathrm{E})$ cases of fertility preservation $(\mathrm{FP})$ by race at our center - White 47O/32E, Black 3O/15E, Asian 13O/7E, and Hispanic 6O/15E $(p<0.001)$. A statistically significant difference in racial distribution by FP type was observed.

Conclusions There is a difference in the observed vs expected racial distribution of patients accessing MIFP. Further studies are needed to identify modifiable factors to better ensure equal opportunity to all patients.
\end{abstract}

Keywords Embryo banking $\cdot$ Fertility preservation $\cdot$ Oocyte cryopreservation $\cdot$ Racial disparity

\section{Introduction}

In 2016, 94 out of every 100,000 women between the ages of 15 and 39 years old were diagnosed with cancer [1], an increase of almost $19 \%$ from the early 2000s [1]. Fortunately, with advances in cancer care, the survival rate of reproductive age cancer patients is also increasing [2]. In fact, the 5-year cancer survival rate was documented as high as $86.4 \%$ in 2011 [1]. In an era where women who choose to reproduce are delaying motherhood until

Paxton E. Voigt

Paxton.Voigt@nyulangone.org

NYU Grossman School of Medicine, New York, NY, USA

2 New York, USA

3 NYU Langone Fertility Center, NYU Langone Health, New York, NY, USA later in life [3], we will see more women diagnosed with cancer before completing, or even beginning, their families. Therefore, fertility preservation (FP) has become a critical component of the multidisciplinary approach to cancer treatment in women of reproductive age. Together, the American Society of Clinical Oncology and the American Society for Reproductive Medicine recommend that providers discuss potential treatment gonadotoxicity and to offer the option of FP to all patients of reproductive age [4, 5].

Oocyte cryopreservation (OC) and embryo banking (EB) are safe and effective methods of preserving one's fertility [6]. Yet, despite these advances in FP services and the wellestablished "frozen hope" that FP provides patients and survivors [7], recent data suggest that fewer than half of women access services [8]. In fact, research has shown the utilization rates to be as low as $4-6 \%[9,10]$. The cause of low utilization rates is likely multifaceted and complex, impacted by an interplay of personal, cultural and/or religious beliefs about life, 
death, cancer, motherhood, as well as ART [11]. In addition, multiple studies have observed sociodemographic and cultural disparities in general infertility care [11-13], yet limited data exist on access disparities in women diagnosed with cancer. It has been suggested that sociodemographic factors may affect utilization of FP services in cancer patients [9]; however, there are conflicting results. Lee and colleagues found that neither race nor ethnicity predicts whether women will utilize FP services before cancer treatment [14]. Whereas, more recently in 2013, Letourneau and colleagues observed that White patients were more likely than non-White patients to seek FP following a cancer diagnosis [9].

Thus, we sought to address this conflict in the literature. Our center is located in one of the most diverse cities in the world. Therefore, we sought to assess whether racial and ethnic disparities impact access to FP services by comparing the racial make-up of women accessing medically indicated FP services at our clinic against the racial make-up of reproductive age women diagnosed with cancer in New York City (NYC).

\section{Materials and methods}

\section{Inclusion/exclusion criteria and subjects}

With IRB approval (\#S13-00389), we performed a retrospective cohort study and cross-sectional comparison of all patients who completed at least one medically indicated OC or EB cycle prior to cancer treatment from January 2017 through December 2018 at the NYU Langone Fertility Center. Patients accessing FP services for a medical indication other than a cancer diagnosis were excluded. The majority of patients seen at our clinic have insurance. The majority are from urban regions of the tri-state area, but our center does draw patients from the surrounding suburban neighborhoods. For broader perspective, the following is the racial breakdown of patients that visited our hospital center in 2018: 47\% White, 13\% Black, $7 \%$ Asian, $23 \%$ other, $8 \%$ not reported [15]. In the same year, $47 \%$ of the total hospital outpatient care were patients with commercial insurance, 33\% had Medicare, 19\% had Medicaid, and $1 \%$ had other insurance or were self-pay [15].

\section{Data collection and measurements}

The electronic medical records for all included subjects were retrospectively reviewed for study variables including age at retrieval, medical indication/diagnosis, type of FP (OC or EB), as well as self-reported race and ethnicity.

The expected incidence of cancer by race in reproductive age women living in NYC was calculated using the most recent cancer incidence data by race from the New York
State Cancer Registry [16, 17], population estimates of reproductive age women living in NYC provided by the New York Department of Health [18], and available NYC census data by race [19]. The calculation is displayed in Fig. 1. This calculation was used to make the statistical comparison of the racial distribution across patients who accessed medically indicated FP services at our center with the racial distribution across reproductive age women who were diagnosed with cancer in NYC.

\section{Analysis}

Statistical analysis included chi-square goodness of fit, test for independence for categorical variables, and Kruskal-Wallis $H$ test for comparisons of our non-parametric continuous variables between racial groups where appropriate. Statistical significance was considered for a $p$ value $<0.05$.

\section{Results}

\section{Demographic data}

Overall, 107 patients were included. There were 64 OC cycles and 43 EB cycles. The average age of all patients in our sample was 32.40 years, with a range of 15-46 years. Table 1 shows the breakdown of age by race. White patients were significantly younger than Black patients (30.6 vs 38.0 years old, $\mathrm{H}-38.52, p=0.036$ ) and those who identified as "other" ( 30.6 vs 41.3 years old, $\mathrm{H}-53.52, p=0.004$ ). The majority of patients were identified as White $(51.4 \%, n=55)$. The rest of the sample were identified as Black $(2.8 \%, n=3)$, Asian $(12.2 \%, n=13)$, Hispanic $(5.6 \%, n=6)$, other $(2.8 \%, n=$ $3)$, or chose not to report $(25.2 \%, n=27)$. Overall, $78.5 \%$ of patients $(n=84)$ had insurance. There was no significant difference in insurance status by race/ethnicity $(p=0.235)$ or by procedure type, OC vs EB ( $p=0.505)$. Overall, $60(56.1 \%)$ patients had a partner at the time of FP. There was no difference in partner status by race/ethnicity $(p=0.076)$. Diagnoses or medical indications were grouped by organ system. Figure 2 shows the breakdown of race by medical indication. The majority were diagnosed with breast cancer $(40.2 \%, n=$ $43)$, followed by gynecologic cancers $(15.0 \%, n=16)$, hematologic cancers $(15.0 \%, n=16)$, neurologic cancers $(5.6 \%, n=$ 6), gastrointestinal cancers $(4.7 \%, n=5)$, sarcomas $(4.7 \%, n=$ 5), endocrine cancers $(2.7 \%, n=4)$, and "other" (skin and nasopharyngeal) $(2.8 \%, n=3)$. The remaining 8 patients (7.5\%) underwent FP for a history of the breast cancer susceptibility syndrome BRCA1/2 with a scheduled bilateral salpingo-oophorectomy. There was no significant difference in racial distribution by cancer type $(p=0.255)$. 


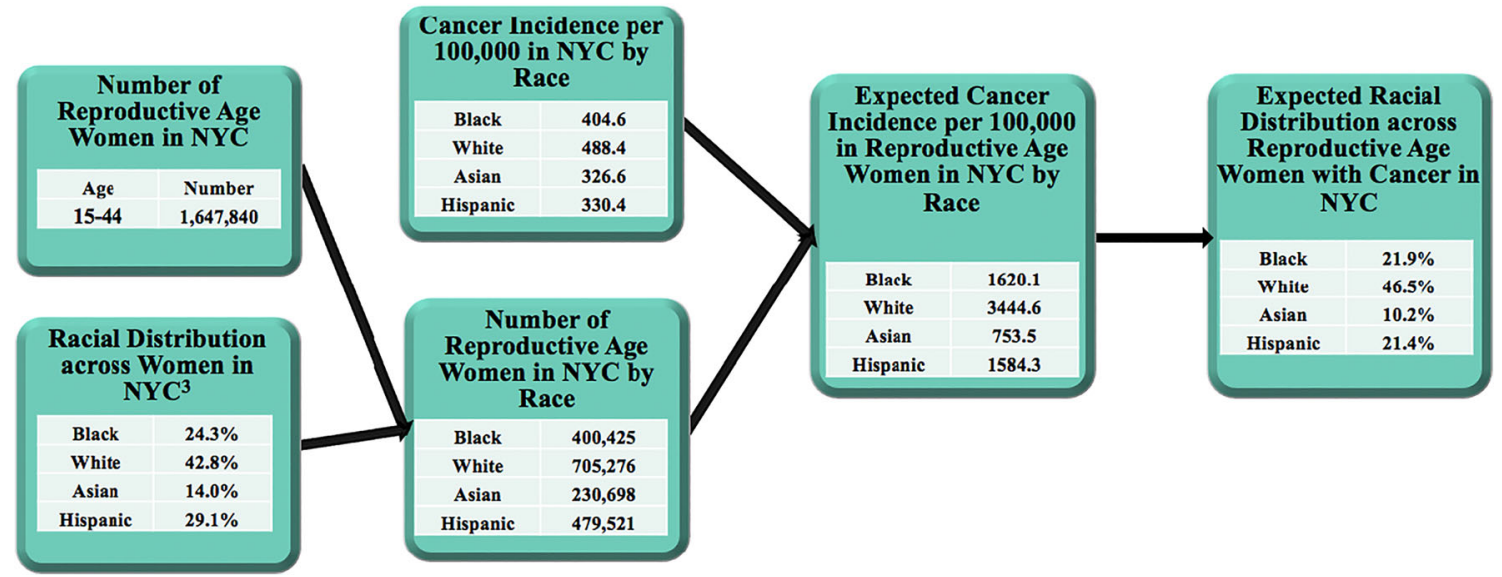

Fig. 1 Calculation of the expected racial distribution across women of reproductive age with cancer in New York City

\section{Comparisons across patients undergoing FP at our clinic to calculated distributions of cancer patients in NYC}

A subgroup analysis was then performed to compare the racial distribution across patients who accessed medically indicated FP services at our clinic against the racial distribution across women of reproductive age diagnosed with cancer in NYC ( $n$ =69). This excluded the BRCA1/2-positive patients and those races not reported by the census [19]. Table 2 shows the demographics of the subgroup by age and FP type. In this subgroup, White patients were overall younger than non-White patients (H 15.32, df 5, $p=0.009)$. The majority of patients $(62.3 \%, n=43)$ had a partner at the time of FP. In this subgroup, there was no statistical significance in partner status by race/ethnicity $\left(X^{2} 6.43\right.$, df $\left.3, p=0.093\right)$. Using the most recent cancer incidence and census data for NYC (Fig. 1), the expected racial distribution across reproductive age women with cancer was calculated to be $42 \%$ White, $21 \%$ Black, $8.9 \%$ Asian, and $28.1 \%$ Hispanic.

A chi-square goodness of fit test was then performed using the calculated "expected" racial distribution compared to the observed rates within the subgroup at our center. A statistically significant difference between observed and expected cases of FP by race at our center was observed (Fig. 3); 47 of our FP patients were White compared to the 32 that were expected, 3

Table 1 Average age of patient by race and ethnicity

\begin{tabular}{lll}
\hline Race/ethnicity & Average age (years) & Total, $n=107(\%)$ \\
\hline White & 30.56 & $55(51.4)$ \\
Black & 38 & $3(2.8)$ \\
Asian & 34.85 & $13(12.1)$ \\
Hispanic & 34 & $6(5.6)$ \\
Other & 41.33 & $3(2.8)$ \\
Not Reported & 33 & $27(25.2)$ \\
\hline
\end{tabular}

were Black compared to 15 expected, 13 were Asian compared to 7 expected, and 6 were Hispanic compared to 15 expected $\left(X^{2} 27.3\right.$, df $\left.3, p<0.001\right)$. This subgroup was further analyzed by FP type (Fig. 4). Overall, 38 patients underwent OC $(55.1 \%)$ and 31 underwent EB (44.9\%). Patients undergoing $\mathrm{OC}$ were significantly younger than those undergoing EB (30.9 vs 34.7 years old, $\mathrm{H} 8.15$, df $1, p=0.004)$. Those undergoing EB were significantly more likely to have a partner than those undergoing OC $\left(X^{2} 34.04\right.$, df $\left.1, p<0.005\right)$. A statistically significant difference in racial distribution by FP type was seen; the majority of White patients underwent OC ( $n=31,66.0 \%$ ), while the majority of non-White patients underwent $\mathrm{EB}(n=2,66.7 \%$ of Black patients, $n=7,53.8 \%$ of Asian patients, and $n=6,100 \%$ of Hispanic patients $)\left(X^{2}\right.$ 10.60, df $3, p<0.014)$.

\section{Discussion}

\section{Representations of cancer patients in the FP population}

Although major technological advances in the field of reproductive medicine have provided cancer survivors with the means to conceive post-treatment, availability of such technologies does not guarantee accessibility to such services, especially for vulnerable populations [20]. This study sought to address a conflict in the literature while also adding to the limited data that exist on the impact of sociodemographic factors on access to medically indicated FP services. Our findings are consistent with those of Letourneau and colleagues, confirming a racial disparity among patients undergoing FP. We showed that following a cancer diagnosis, Black and Hispanic women were less likely to utilize FP services in our clinic than White and Asian patients. Furthermore, this study elucidated an association between race and utilization of FP service type, OC or EB. In our study, White patients 
Fig. 2 Medical indication for fertility reservation by race and ethnicity

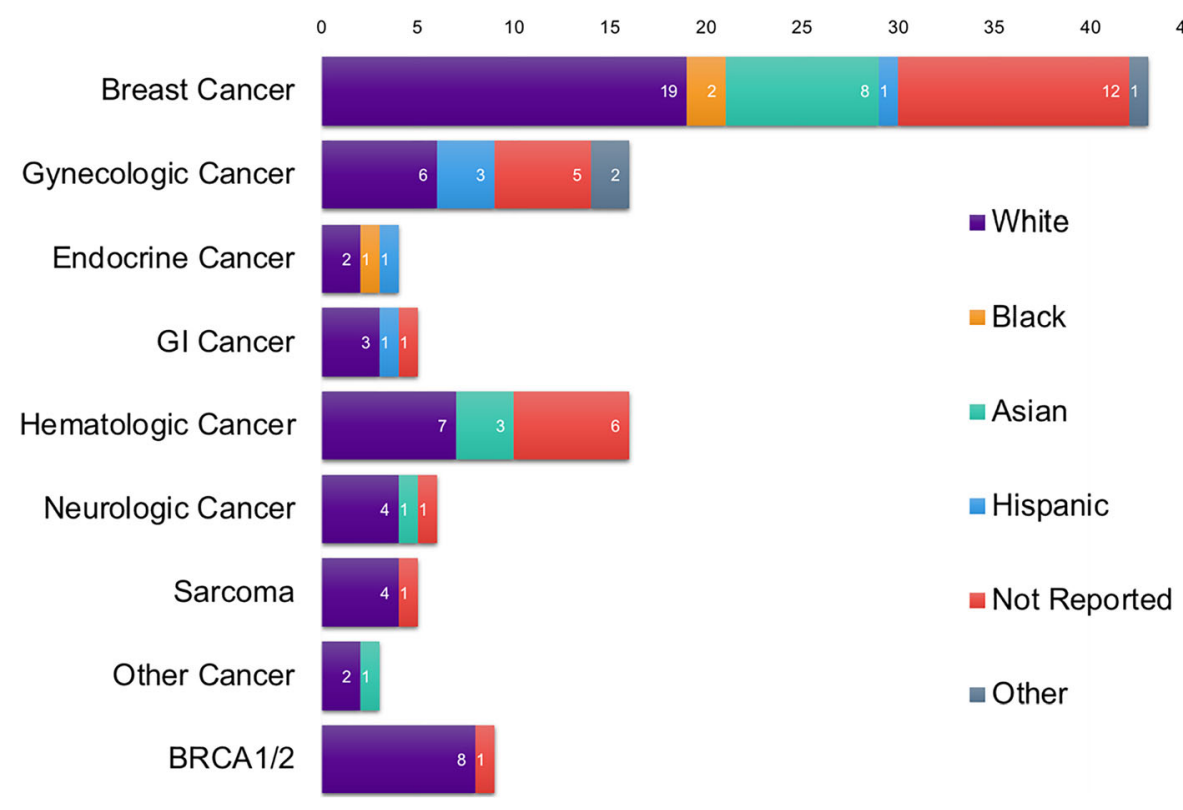

were more likely to undergo OC and non-White patients were more likely to undergo EB. All Black and Hispanic patients accessing FP had a partner. While further studies are needed to determine possible explanations for this finding, it suggests that non-White patients were more likely to access FP services when they had a partner with whom they knew they wanted to procreate. Across all races, the average age for patients who underwent $\mathrm{EB}$ was higher. It is consistent with what one would expect, as younger patients are less likely than older patients to have a partner with whom they would want to procreate. It did not go unnoticed, however, that, within the subgroup analysis, White patients were overall younger than non-White patients, regardless of FP type. This finding stresses the need for further study on its significance, to ensure that all patients have equal access to FP services, regardless of age, race, or having a partner.

\section{Socioeconomic impacts}

We would be remiss not to discuss the cost and potential financial burden of FP. The majority of patients in this study were insured, which may limit our generalizability. However, racial disparities in access to FP were still seen despite no difference in insurance status by race, ethnicity, or procedure type. These findings are consistent with racial disparities seen in general infertility care. Jain and colleagues showed that even in MA, a state with a comprehensive mandate to cover infertility treatment, significant disparities exist. Black and Hispanic women were shown to underutilize infertility services, while the majority of women accessing such services were White, highly educated, and wealthy [21, 22]. Given that FP is not yet routinely covered by insurance and that the costs of FP is significant [23], we recognize the possibility that one's household income would impact one's ability to access FP [9]. However, Letourneau and colleagues found no association between income and FP access [9], and Lee and colleagues found no association between income and whether women accessed FP vs post-treatment fertility services [14].

Our center is a private clinic, which may again limit the generalizability. However, most public fertility clinics, such as our center's affiliation with the safety net hospital Bellevue Hospital Center, are not able to offer FP services. Cancer patients seen at Bellevue are referred to our center for care [24]. The cost of FP has often been cited as a barrier to access $[25,26]$, but our center, as many do, utilizes outside programs to offer assistance to our patients. "Sharing Hope" through the
Table 2 Demographics of subgroup analysis

\begin{tabular}{lllllll}
\hline Race & $\begin{array}{l}\text { Average age } \\
\text { (years) }\end{array}$ & $\begin{array}{l}\text { Age OC } \\
\text { (years) }\end{array}$ & $\begin{array}{l}\text { OC (years) } \\
(\%)\end{array}$ & $\begin{array}{l}\text { Average age EB } \\
\text { (years) }\end{array}$ & $\begin{array}{l}\text { EB (years) } \\
(\%)\end{array}$ & Total \\
\hline White & 30.11 & 28.87 & $31(66.0)$ & 32.5 & $16(34.0)$ & 47 \\
Black & 38 & 34 & $1(33.33)$ & 40 & $2(66.67)$ & 3 \\
Asian & 34.85 & 32.83 & $6(46.15)$ & 36.57 & $7(53.85)$ & 13 \\
Hispanic & -- & 0 & $0(0.0)$ & 34 & $6(75.0)$ & 6 \\
& & 29.63 & 38 & 34.19 & 31 & 69 \\
\hline
\end{tabular}


Fig. 3 Observed vs expected fertility preservation cases by race and ethnicity

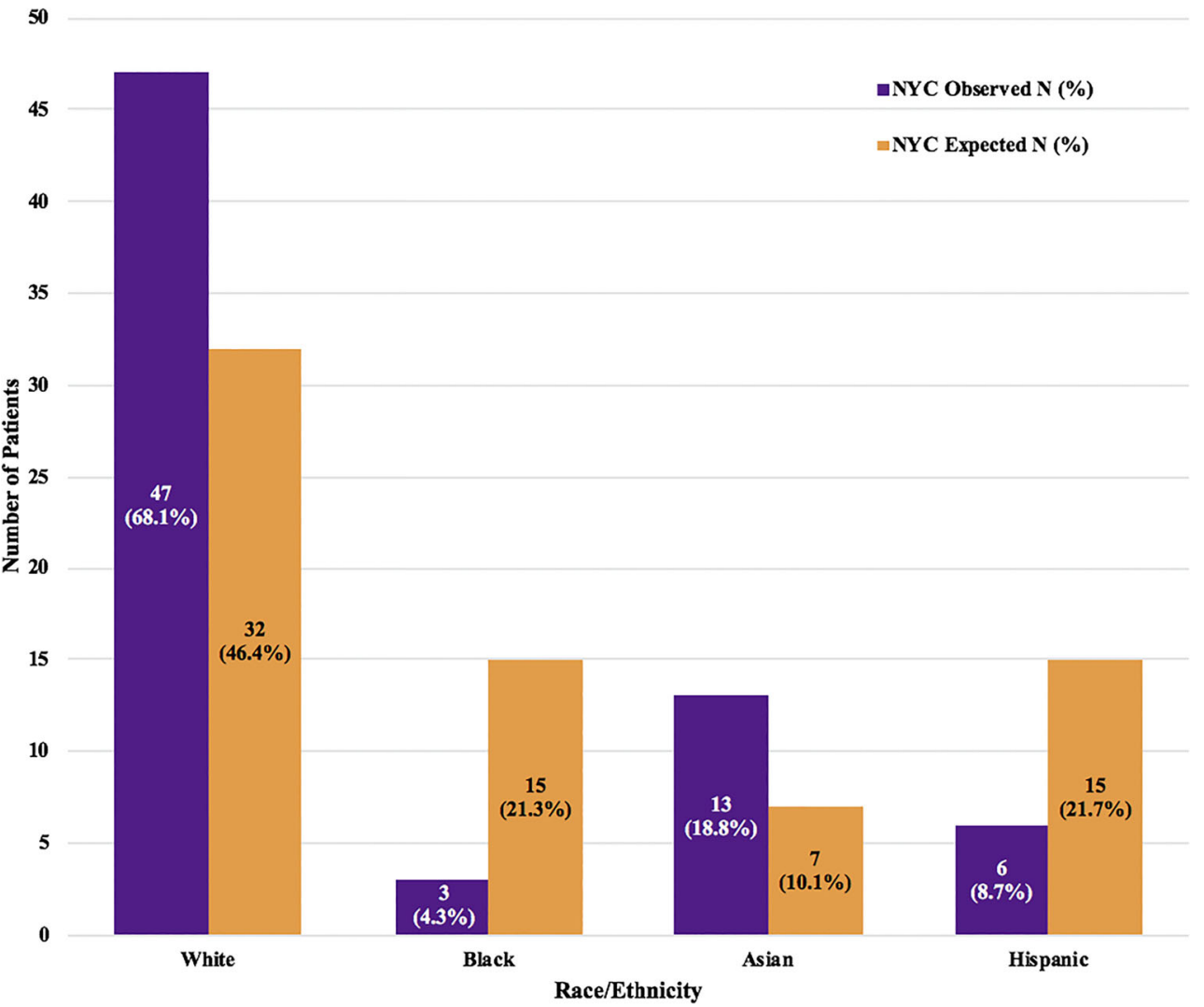

Livestrong Foundation provides assistance with medication to eligible patients diagnosed with cancer [27]. Additionally, Ferring Pharmaceuticals' Heart Beat Program provides fertility medications free of cost to women who have been diagnosed with cancer. Yet, with recent studies estimating that female patients eligible for financial assistance through the Livestrong foundation would still be responsible for paying between $\$ 3000$ and $\$ 7000$ [27], these programs do not eliminate cost as a barrier to access.

In March of 2019, the NY State Legislature passed the Fertility Preservation Bill, making New York the 6th state in the USA to mandate FP coverage. Finalized in the state's 2020 budget, the bill requires coverage of all medically necessary FP procedures by all commercial insurers [28]. Research has shown that state-mandated insurance coverage increases the utilization of infertility services approximately 3 -fold [26]. Therefore, a follow-up study is intended to compare the results of this study to data collected in the 2020 calendar year to determine whether this advance in legislation helped to expand access across all races.

\section{Cultural influences}

While mandated insurance benefits may enhance accessibility to FP services, they are not sufficient in realizing equal access for all women. One must also consider the additional personal, cultural, and/or religious beliefs about life, death, cancer, motherhood, and ART, which may impact the type of FP procedure a patient chooses, if at all [11]. Ethnic minorities have been found to express more concerns than White women about the social stigma of infertility and using science or technology to conceive [29]. Moreover, Black women have been shown to be more concerned than White women about the potential for poor pregnancy outcomes in the setting of ART [11]. Extending beyond one's race and ethnicity, some religious groups oppose the creation of embryos due to the impossibility to discard or donate early embryos to research, which may affect one's choice of FP type [30]. Furthermore, the overwhelming emotion and uncertainty that comes with a new cancer diagnosis may contribute to the complexity of the decision [31]. Despite patients expressing interest in pursuing FP following a cancer diagnosis, a much lower majority were willing to delay treatment in order to do so [32]. Finally, age at first motherhood, which may be different between racial and ethnic groups, may also impact the need for FP. In 2015, the majority of babies born to Hispanic $(56.46 \%)$ and Black mothers $(50.31 \%)$ in NYC was born to women younger than 30 years old, compared with the majority of Asian/Pacific Islander $(60.00 \%)$ and White $(66.07 \%)$ mothers who were older than 30 years (Fig. 4) [33]. Previous studies have shown that women who access counseling and FP services are more likely to be childless at the time of their treatment $[34,35]$. Thus, it is possible that racial and ethnic differences in parity influence utilization of FP services. We acknowledge that the 

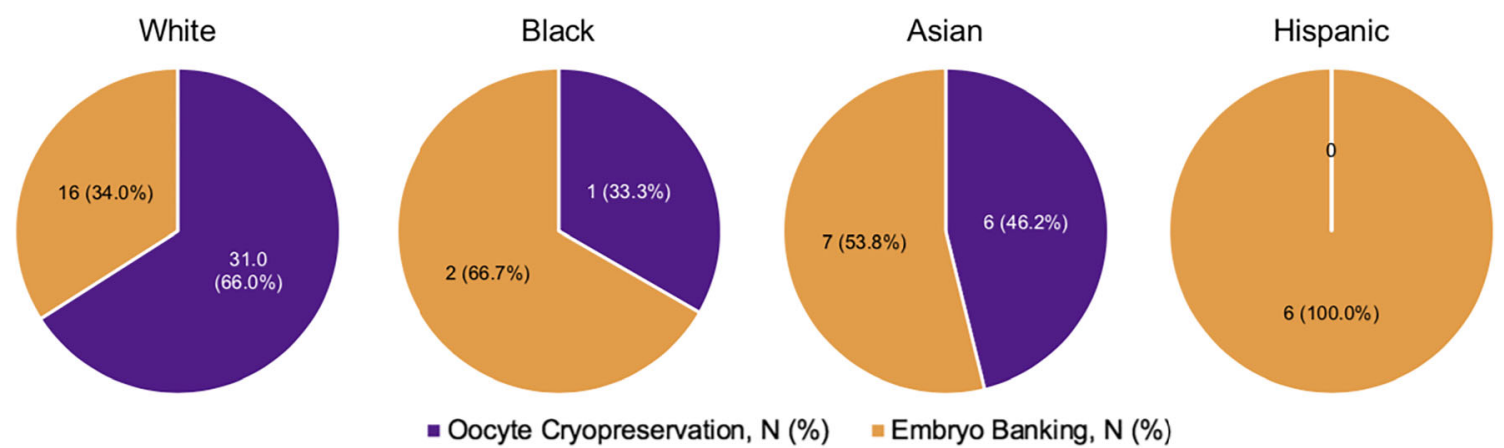

Fig 4 Subgroup analysis. Fertility preservation cycle type by race and ethnicity

not including parity as a covariate in this analysis is an important limitation of this study. However, parity, while important, may not be the best surrogate marker for one's family building choices. A woman's decision for future fertility is both personal and complex, and likely interwoven with many cultural and religious influences, thus highlighting the need for further research on reasons why women choose for or against pursuing FP.

\section{Strengths and weaknesses}

This study has several strengths. It is unique in our utilization of a mathematical process to assess for a racial disparity in access to FP services in women diagnosed with cancer. Additionally, our findings contribute further context about people who access both oocyte cryopreservation and embryo cryopreservation. Understanding the differences between these two subpopulations (by FP type) may be helpful for patient counseling and for improving referral patterns from oncologists.

However, there are several limitations. First, the cancer incidence used in the calculation of the expected racial make-up of the patient population was the incidence of all cancer types. Yet not all cancers require treatment with gonadotoxic therapy, therefore possibly overestimating the number of women who would need to access medically indicated FP services. However, this overestimation was held constant across all racial groups, and therefore should not affect the overall racial distribution and the calculated expected number of patients by race. Second, the use of self-reported race on demographic forms invites the possibility of selection bias. Moreover, it excludes the 27 patients who chose to not report their race/ethnicity. We could extrapolate that these patients would also not report on the census, but we cannot ignore the fact that had they reported, the data might have been skewed differently. While the fill-in-the-blank format of our clinic's demographic form promotes inclusivity, it does exclude the patients who may not self-identify as one of the four races reported by the Census Bureau. This limitation highlights an issue that extends beyond the scope of this study and emphasizes the need for more diverse and inclusive measures of access to care in order to better investigate and address racial disparities in access to healthcare as a whole. Future research is needed to develop a more inclusive measure that more adequately reflects the diversity of today's world. A further limitation is that our clinic does not offer ovarian tissue cryopreservation (OTC) for FP at this time, which may affect how patients choose their center for care. As such, an analysis of patients who are candidates of OTC needs to be addressed. Lastly, we did not have access to all referred patients, just those seen at our clinic. While referral rate may not be an accurate surrogate marker for FP utilization, evidenced by a previous study which showed a $4 \%$ FP utilization rate despite a $61 \%$ referral rate [9], we recognize that evaluating all referred patients may provide further insight into whether the data presented represent a disparity in access to care or a tendency to present to our center vs another for cancer care. Therefore, missing this referral information is a major weakness of our study. Moreover, while our center is located in the heart of one of the most diverse cities in the USA, it is not the only fertility clinic in NYC. A patient's choice of which clinic to visit has been shown to be determined by a complex interplay between patient and provider characteristics, including race and ethnicity $[33,34]$, and future studies should compare the racial make-up of multiple fertility centers across NYC. Moreover, this study highlights the need for further research into identifying potential points of intervention and modifiable solutions to rectify this disparity in access. Considerations include more inclusive (language, representation) educational materials, increased access to dedicated liaisons between oncologists and fertility centers with knowledge of cost support programs, better education for language interpreters, increasing support groups with a focus on cultural nuances. This study is important in that it serves as a baseline to which future studies evaluating strategies to further improve uptake among racial/ethnic minorities can be compared.

\section{Timely implications}

It is important to note that at the time of submission of this manuscript, the USA was in the midst of the COVID-19 pandemic. Early data suggest a racial disparity in COVID-19 cases [36]. In accordance with an Emergency Executive 
Order [37], all ART cycles at our center were paused for safety with the exclusion of FP patients who were able to continue oocyte cryopreservation. We highlight these points to draw attention to the need for future studies to determine how COVID-19 impacted minority populations undergoing FP and/or fertility treatment.

\section{Conclusions}

In conclusion, we have shown that the racial distribution across patients accessing medically necessary FP services at our center did not reflect the expected racial distribution across reproductive age women diagnosed with cancer in NYC. There was an observed difference in the racial make-up of patients pursuing OC vs EB. Overall, Black and Hispanic patients were underrepresented in FP and among non-White patients there was a higher incidence of EB than OC. Further studies are needed to determine if these differences generalize beyond our clinic and to identify modifiable factors, such as patients' socioeconomic status and outreach and education opportunities, along with the consideration of cultural, religious, and financial barriers, to ensure equal opportunity to all patients.

Acknowledgments The authors gratefully acknowledge the patients who chose to seek care at the NYU Langone Fertility Clinic.

\section{References}

1. SEER*Explorer: an interactive website for SEER cancer statistics; 2019. [cited 2019 November 15]; Available from: https://seer. cancer.gov/explorer/.

2. Siegel RL, Miller KD, Jemal A. Cancer statistics, 2016. CA Cancer J Clin. 2016;66(1):7-30.

3. Bellieni C. The best age for pregnancy and undue pressures. J Family Reprod Health. 2016;10(3):104-7.

4. Lee SJ, et al. American Society of Clinical Oncology recommendations on fertility preservation in cancer patients. J Clin Oncol. 2006;24(18):2917-31.

5. Fertility preservation and reproduction in patients facing gonadotoxic therapies: a committee opinion. Fertil Steril. 2013;100(5):1224-31.

6. Fertility preservation and reproduction in patients facing gonadotoxic therapies: an Ethics Committee opinion. Fertil Steril. 2018;110(3):380-6.

7. Quinn GP, Vadaparampil ST, Jacobsen PB, Knapp C, Keefe DL, Bell GE, et al. Frozen hope: fertility preservation for women with cancer. J Midwifery Womens Health. 2010;55(2):175-80.

8. Lawson AK, McGuire JM, Noncent E, Olivieri JF Jr, Smith KN, Marsh EE. Disparities in counseling female cancer patients for fertility preservation. J Women's Health (Larchmt). 2017;26(8):88691.

9. Letourneau JM, Smith JF, Ebbel EE, Craig A, Katz PP, Cedars MI, et al. Racial, socioeconomic, and demographic disparities in access to fertility preservation in young women diagnosed with cancer. Cancer. 2012;118(18):4579-88.
10. Selter $\mathrm{J}$, et al. Use of fertility preservation services in female reproductive-aged cancer patients. Am J Obstet Gynecol. 2019;221(4):328.e1-328.e16.

11. Missmer SA, Seifer DB, Jain T. Cultural factors contributing to health care disparities among patients with infertility in Midwestern United States. Fertil Steril. 2011;95(6):1943-9.

12. Chandra A, Copen CE, Stephen EH. Infertility service use in the United States: data from the National Survey of Family Growth, 1982-2010. Natl Health Stat Report. 2014;(73):1-21.

13. Bitler M, Schmidt L. Health disparities and infertility: impacts of state-level insurance mandates. Fertil Steril. 2006;85(4):858-65.

14. Lee S, Heytens E, Moy F, Ozkavukcu S, Oktay K. Determinants of access to fertility preservation in women with breast cancer. Fertil Steril. 2011;95(6):1932-6.

15. NYU Langone Health in the Community: 2017-2018. NYU Langone Health. p. 25. Retrieved October 20, 2020, from https:// nyulangone.org/files/nyulangone-health-in-the-community-report2019.pdf.

16. Cancer Incidence and Mortality among Asians and Pacific Islanders, New York City, 2012-2016. New York State Department of Health; 2016. Retrieved October 20, 2020 from: https://www.health.ny.gov/statistics/cancer/registry/table4/ tb4asiannyc.htm.

17. Cancer by Ethnicity (Hispanic Origin). New York State Department of Heatlh; 2016. Retrieved October 20, 2020 from: https://www. health.ny.gov/statistics/cancer/registry/table5.htm.

18. Estimated Population by Age, Sex and Region. New York State Department of Health; 2011. Retrieved October 20, 2020 from: https://www.health.ny.gov/statistics/vital_statistics/2010/table01. $\mathrm{htm}$.

19. Quick Facts: New York City, New York; 2018. Available from: https://www.census.gov/quickfacts/fact/table/ newyorkcitynewyork/SEX255218.

20. Gulliford M, Figueroa-Munoz J, Morgan M, Hughes D, Gibson B, Beech R, et al. What does 'access to health care' mean? J Health Serv Res Policy. 2002;7(3):186-8.

21. Jain T. Socioeconomic and racial disparities among infertility patients seeking care. Fertil Steril. 2006;85(4):876-81.

22. Jain T, Hornstein MD. Disparities in access to infertility services in a state with mandated insurance coverage. Fertil Steril. 2005;84(1): 221-3.

23. Quinn GP, Vadaparampil ST, McGowan Lowrey K, Eidson S, Knapp C, Bukulmez O. State laws and regulations addressing third-party reimbursement for infertility treatment: implications for cancer survivors. Fertil Steril. 2011;95(1):72-8.

24. Blakemore JK, Maxwell SM, Hodes-Wertz B, Goldman KN. Access to infertility care in a low-resource setting: bridging the gap through resident and fellow education in a New York City public hospital. J Assist Reprod Genet. 2020;37:1545-52.

25. Flink DM, Sheeder J, Kondapalli LA. A review of the oncology patient's challenges for utilizing fertility preservation services. J Adolesc Young Adult Oncol. 2017;6(1):31-44.

26. Jain T, Harlow BL, Hornstein MD. Insurance coverage and outcomes of in vitro fertilization. N Engl J Med. 2002;347(9):661-6.

27. Omesi L, Narayan A, Reinecke J, Schear R, Levine J. Financial assistance for fertility preservation among adolescent and young adult cancer patients: a utilization review of the sharing Hope/ LIVESTRONG Fertility Financial Assistance Program. J Adolesc Young Adult Oncol. 2019;8(5):554-9.

28. State Legislation: New York; 2019. [cited 2019 October 30]; Available from: https://www.allianceforfertilitypreservation.org/ state-legislation/new-york.

29. Humphries LA, et al. Influence of race and ethnicity on in vitro fertilization outcomes: systematic review. Am J Obstet Gynecol. 2016;214(2):212.e1-212.e17. 
30. Sallam HN, Sallam NH. Religious aspects of assisted reproduction. Facts Views Vis ObGyn. 2016;8(1):33-48.

31. Kangas M, Henry JL, Bryant RA. A prospective study of autobiographical memory and posttraumatic stress disorder following cancer. J Consult Clin Psychol. 2005;73(2):293-9.

32. Burns KC, Boudreau C, Panepinto JA. Attitudes regarding fertility preservation in female adolescent cancer patients. J Pediatr Hematol Oncol. 2006;28(6):350-4.

33. Birth and Death. 2015. April 11, 2020]; Available from: https:// a816-health.nyc.gov/hdi/epiquery/visualizations?PageType= ts \&PopulationSource $=$ Birth \&Topic $=8 \&$ Subtopic $=38$.

34. Lawrenz B, Jauckus J, Kupka MS, Strowitzki T, von Wolff M. Fertility preservation in $>1,000$ patients: patient's characteristics, spectrum, efficacy and risks of applied preservation techniques. Arch Gynecol Obstet. 2011;283(3):651-6.
35. Klock SC, Zhang JX, Kazer RR. Fertility preservation for female cancer patients: early clinical experience. Fertil Steril. 2010;94(1): $149-55$.

36. Aubrey A. CDC hospital data point to racial disparity in covid-19 cases. April 8, 2020. April 9, 2019]; Available from: https://www. npr.org/sections/coronavirus-live-updates/2020/04/08/830030932/ cdc-hospital-data-point-to-racial-disparity-in-covid-19-cases.

37. de Blasio M. NYC health + hospitals and bioreference laboratories announce expanded capacity to allow 5,000 daily COVID-19 tests. MArch 17, 2020. April 9, 2020]; Available from: https://www1. nyc.gov/office-of-the-mayor/news/160-20/mayor-de-blasio-nychealth-hospitals-bioreference-laboratories-expanded-capacity-to.

Publisher's note Springer Nature remains neutral with regard to jurisdictional claims in published maps and institutional affiliations. 